\title{
CORRESPONDENCE
}

\section{Natural course of sleep disordered breathing after acute myocardial infarction}

\section{To the Editor:}

In a recent issue of the European Respiratory Journal, BUCHNER et al. [1] reported an alleviation of sleep apnoea in 40 patients with acute myocardial infarction (AMI) 12 weeks after the event, associated with an improvement of $\geqslant 5 \%$ in the ejection fraction (EF). Moderate to severe sleep apnoea was observed in $55 \%$ of the patients. At the second evaluation the respiratory events were reduced significantly more in the improved EF group. The authors' novel observation was that the decrease in the apnoea-hypopnoea index (AHI) was the result of a reduction in obstructive, but not central respiratory events.

Our own study of the time course of sleep apnoea after a first AMI was published several months earlier in the Journal of Clinical Sleep Medicine [2], although it was not cited by BUCHNER et al. [1]. The study included 52 patients with first-ever acute coronary syndrome (ACS) who underwent polysomnography (PSG) on the third day after the acute cardiac event and were re-evaluated with PSG 1 and 6 months later. We observed a progressive decrease in the AHI over time, which was due to the reduction of obstructive respiratory events while central events remained unchanged. This was a novel observation that contrasted with previous studies [3].

There are some concerns related to the important study by BUCHNER et al. [1] that must be addressed.

First, the differentiation into two groups based on a $\geqslant 5 \%$ improvement in EF. This cut-off was used by CINTRON et al. [4] in 1993, who found that patients with an improvement in EF of $\geqslant 5 \%$ had lower cardiac event rates over 2 years. However, as those authors stressed, the left ventricular (LV) EF is only a crude index. In addition, their patients had an EF around 30\%, much lower than in both the study BUCHNER et al. [1] and our own [2]. JosHi et al. [5], in a more recent study, found that an $8 \%$ threshold in EF improvement was associated with better survival. This threshold was twice the mean inter-observer difference in EF measurements and ensured that patients with such an improvement truly had significant increases in global LV systolic function. The design of the study carried out by BUCHNER et al. [1], splitting the patient population into two small groups using a questionable threshold for EF improvement that was based on a study dating from 20 years ago, decreases the statistical power of the study. In contrast to the above, the 52 patients in our study had a preserved LVEF that was statistically unchanged during the follow-up period and could not be used to discriminate the patients into different groups. Based on the data reported by BUCHNER et al. [1], the entire patient population showed only a modest, statistically nonsignificant reduction in sleep apnoea. By contrast, the data from our study showed a clear improvement in the assessed $\mathrm{AHI}$ at both selected follow-up time points [2].

The second concern relates to the low reported sleep efficiency. There are no other data related to the total sleep time, wake after sleep onset, sleep latency and, especially, arousal index. The low sleep efficiency may be related to the well-known first-night effect, even though this should have been eliminated at least in the follow-up PSG. The absence of data related to the arousal index might be crucial, since the influence of this factor on sleep micro-architecture and the ensuing respiratory instability may predispose to the occurrence of respiratory events. In our study, although the sleep efficiency 3 days after the acute event was low, it increased significantly in the follow-up PSGs [2].

Thirdly, sleep position and sleep stage can influence the frequency of obstructive events. Our study, contrary to that of BUCHNER et al. [1], provides detailed data related to the AHI during supine and non-supine sleep, as well as during rapid eye movement (REM) and non-REM sleep.

Fourthly, body weight is of importance, and the improved or stable EF groups defined by BUCHNER et al. [1] were different in this respect. A trend was reported where the improved EF group showed a weight loss, whereas the unchanged EF group gained weight. This, taken together with the absence of sleep position data and the small number of subjects, significantly decreases the accuracy of the given results. In our study, body weight showed no statistically significant changes during the follow-up period.

Both studies showed a high incidence of sleep apnoea after the acute cardiac event, with significant improvement over time. The main issue is not the avoidance of unjustified long-term continuous positive airway pressure when the severity of sleep apnoea is likely to improve, but the necessary actions immediately after a possibly life-threatening cardiac event. Patients with ACS have a high risk of sleep apnoea and need for treatment. However, PSG is expensive, with multiple technical difficulties, and cannot be advised for all subjects. Nevertheless, a $>50 \%$ risk of sleep apnoea cannot be overlooked, and is sufficient to justify seeking an evaluation by a sleep specialist.

\section{Charalampos Mermigkis, Izolde Bouloukaki and Sophia E. Schiza \\ Sleep Disorders Unit, Dept of Thoracic Medicine, University General Hospital, Medical School of the University of Crete, Heraklion, Greece.}


Correspondence: S.E. Schiza, Sleep Disorders Unit, Dept of Thoracic Medicine, University of Crete, 71110 Heraklion, Crete, Greece. E-mail: schiza@med.uoc.gr

Statement of Interest: None declared.

\section{REFERENCES}

1 Buchner S, Greimel T, Hetzenecker A, et al. Natural course of sleepdisordered breathing after acute myocardial infarction. Eur Respir $J$ 2012; 40: 1173-1179.

2 Schiza SE, Simantirakis E, Bouloukaki I, et al. Sleep disordered breathing in patients with acute coronary syndromes. J Clin Sleep Med 2012; 8: 21-26.
3 BaHammam A, Al-Mobeireek A, Al-Nozha M, et al. Behaviour and time-course of sleep disordered breathing in patients with acute coronary syndromes. Int J Clin Pract 2005; 59: 874-880.

4 Cintron G, Johnson G, Francis G, et al. Prognostic significance of serial changes in left ventricular ejection fraction in patients with congestive heart failure. The V-HeFT VA Cooperative Studies Group. Circulation 1993; 87: Suppl. 6, VI17-VI23.

5 Joshi K, Alam I, Ruden E, et al. Effect of improvement in left ventricular ejection fraction on long-term survival in revascularized patients with ischaemic left ventricular systolic dysfunction. Eur J Echocardiogr 2011; 12: 454-460. 